\title{
NATURAL CONVECTION IN A SQUARE CAVITY WITH PARTIALLY ACTIVE VERTICAL WALLS: TIME-PERIODIC BOUNDARY CONDITION
}

\author{
N. NITHYADEVI, P. KANDASWAMY, AND S. SIVASANKARAN
}

Received 27 January 2006; Revised 15 May 2006; Accepted 22 May 2006

A numerical study of transient natural convection in a square cavity with partly thermally active side walls is introduced. The thermally active regions of the side walls are periodic in time. Top and bottom of the cavity are adiabatic. Nine different positions of the thermally active zones are considered. The governing equations are solved using control volume method with power-law scheme. The results are obtained for various values of amplitude, period, and Grashof numbers ranging from $10^{4}-10^{6}$ and different thermally active situations. It is found that the average heat transfer increases by increasing amplitude for $P=1,5$, and decreasing for $P=3$. The average Nusselt number behaves nonlinearly as a function of period.

Copyright (c) 2006 N. Nithyadevi et al. This is an open access article distributed under the Creative Commons Attribution License, which permits unrestricted use, distribution, and reproduction in any medium, provided the original work is properly cited.

\section{Introduction}

Natural convection in fluid-filled rectangular enclosures has received considerable attention over the past several years due to the wide variety of applications that involve natural convection processes. These applications span such diverse fields as solar energy collection, nuclear reactor operation and safety, the energy efficient design of building, room, and machinery, waste disposal, and fire prevention and safety. The oscillation-induced heat transport has been studied by a number of researchers due to its many industrial applications, such as bioengineering, chemical engineering, and so forth.

Antohe and Lage [1] studied the amplitude effect on convection induced by timeperiodic heating. It is shown that the convection intensity within the enclosure increases linearly with heating amplitude. Crunkleton et al. [2] studied the numerical simulation of periodic flow oscillation for low Prandtl number fluids in rectangular enclosure. They observed nonperiodic flows for rectangular cavity with aspect ratio 2.0. Kim et al. [3] studied the buoyant convection with internal heat generation under oscillating sidewall temperature of a cavity. It is found that the secondary peak resonance is detected for higher internal Rayleigh number. 
Kuhn and Oosthuizen [4] numerically studied unsteady natural convection in a partially heated rectangular cavity. They concluded that as the heated location moves from the top to the bottom, the Nusselt number increases upto a maximum and then decreases. Lakhal et al. [5] studied the transient natural convection in a square cavity partially heated from side. In the first, the temperature is varied sinusoidally with time while in the second, it varies with a pulsating manner. The results showed that the mean values of heat transfer and flow intensity are considerably different with those obtained in stationary regime.

Pantokratoras [6] studied natural convection of water near the density extremum along a vertical plate with sinusoidal surface temperature variation. It is found that there is an inner boundary layer near the plate with periodic characteristics. Saeid and Mohamad [8] studied a nonequilibrium model of periodic free convection and discussion. Their results show that increasing the amplitude and frequency of the oscillating surface temperature decreases the heat transfer rate.

Most of the above-cited works are concerned with natural convection in rectangular geometries due to either a vertically or horizontally imposed heat flux or temperature difference. In fields like solar energy collection and cooling of electronic components, the active walls may be subject to abrupt temperature nonuniformities due to shading or other effects. The relative position of the hot and cold wall regions has significant effects on the flow pattern and heat transfer. This paper describes the natural convection in a square cavity with periodically oscillating partly thermally active vertical walls for nine different locations.

\section{Mathematical formulation}

Consider a two-dimensional square cavity of length $L$ filled with a fluid as shown in Figure 2.1. A portion of the right side wall is kept at a temperature $\theta_{c}$ and a portion of the left side wall temperature is periodic in time. The remaining parts of the cavity are insulated. For nine different combination of thermally active wall locations, the heat transfer will be investigated.

Under these assumptions the equations governing the motion of a two-dimensional viscous incompressible fluid may be written as

$$
\begin{gathered}
\frac{\partial u}{\partial x}+\frac{\partial v}{\partial y}=0 \\
\frac{\partial u}{\partial t}+u \frac{\partial u}{\partial x}+v \frac{\partial u}{\partial y}=-\frac{1}{\rho_{0}} \frac{\partial p}{\partial x}+\nu \nabla^{2} u-\frac{\rho}{\rho_{0}} g \\
\frac{\partial v}{\partial t}+u \frac{\partial v}{\partial x}+v \frac{\partial v}{\partial y}=-\frac{1}{\rho_{0}} \frac{\partial p}{\partial y}+v \nabla^{2} v \\
\frac{\partial \theta}{\partial t}+u \frac{\partial \theta}{\partial x}+v \frac{\partial \theta}{\partial y}=\alpha \nabla^{2} \theta
\end{gathered}
$$

where

$$
\rho=\rho_{0}\left[1-\beta\left(\theta-\theta_{c}\right)\right] .
$$


N. Nithyadevi et al. 3

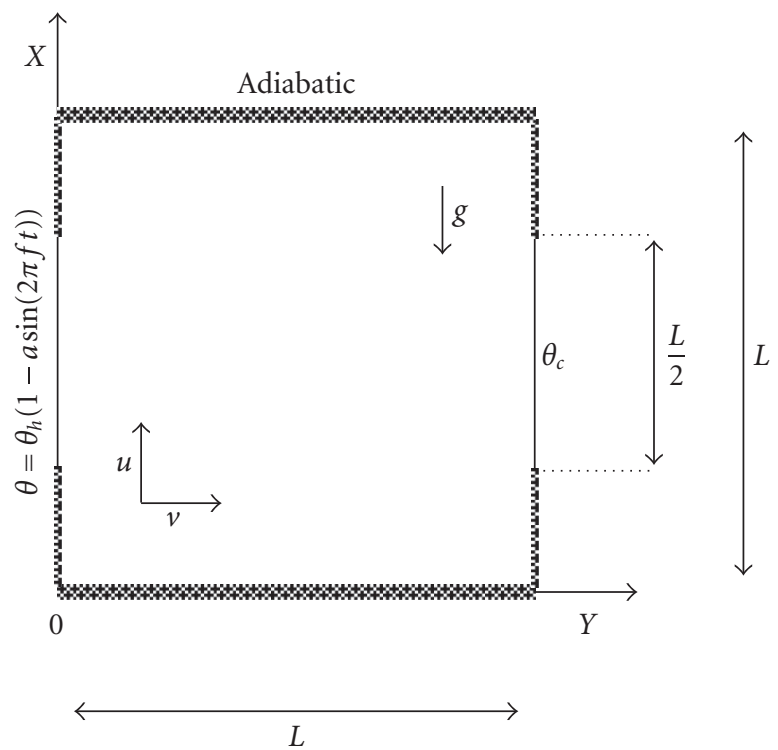

Figure 2.1. Physical configuration.

The appropriate initial and boundary conditions are

$$
\begin{gathered}
t=0 ; \quad u=v=0, \quad \theta=\theta_{c}, \\
t>0 ; \quad u=v=0 \quad \text { on all walls, } \\
\theta=\theta_{h}(1-a \sin (2 \pi f t)) \quad \text { hot part, } \quad \theta=\theta_{c} \quad \text { cold part, } \\
\frac{\partial \theta}{\partial n}=0 \quad \text { elsewhere. }
\end{gathered}
$$

Introducing the following nondimensional variables: $\tau=t /\left(L^{2} / \nu\right),(X, Y)=(x, y) / L$, $f=1 / p, P=p \nu / L^{2}, A=\left(a / \theta_{h}\right) \theta_{c},(U, V)=(u, v) /(\nu / L), \Psi=\psi / \nu, \zeta=\omega /\left(\nu / L^{2}\right), T=$ $\left(\theta-\theta_{c}\right) /\left(\theta_{h}-\theta_{c}\right)$, with $\theta_{h}>\theta_{c}$, we get the vorticity-stream function formulation of the above problem (2.1) as

$$
\begin{gathered}
\frac{\partial T}{\partial \tau}+U \frac{\partial T}{\partial X}+V \frac{\partial T}{\partial Y}=\frac{1}{\operatorname{Pr}} \nabla^{2} T \\
\frac{\partial \zeta}{\partial \tau}+U \frac{\partial \zeta}{\partial X}+V \frac{\partial \zeta}{\partial Y}=\nabla^{2} \zeta+\mathrm{Gr} \frac{\partial T}{\partial Y} \\
\nabla^{2} \Psi=-\zeta
\end{gathered}
$$


4 Convection in cavities with partially active walls

where

$$
U=-\frac{\partial \Psi}{\partial Y}, \quad V=\frac{\partial \Psi}{\partial X}, \quad \zeta=\frac{\partial U}{\partial Y}-\frac{\partial V}{\partial X}
$$

The initial and boundary conditions in the dimensionless form are

$$
\begin{gathered}
\tau=0 ; \quad \Psi=0, \quad T=0, \\
\tau>0 ; \quad \Psi=\frac{\partial \Psi}{\partial Y}=0, \quad \frac{\partial T}{\partial X}=0, \quad \text { at } X=0 \& 1, \\
T=1-A \sin \frac{\pi \tau}{P} \quad \text { hot part, } \quad T=0 \quad \text { cold part at } Y=0 \& 1, \\
\frac{\partial T}{\partial n}=0 \quad \text { elsewhere at } Y=0 \& 1 .
\end{gathered}
$$

Nondimensional parameters that appear in the equations are $\mathrm{Gr}=g \beta\left(\theta_{h}-\theta_{c}\right) L^{3} / \nu^{2}$ Grashof number, $\operatorname{Pr}=v / \alpha$ Prandtl number, $g$ acceleration due to gravity, $v$ kinematic viscosity, $\alpha$ thermal diffusivity, $\beta$ coefficient of thermal expansion, $T$ dimensionless temperature, $t$ time, $\theta$ temperature, $A$ amplitude, $2 P$ period. The local Nusselt number is defined by $\mathrm{Nu}=\partial T /\left.\partial Y\right|_{y=0}$ resulting in the average Nusselt number as $\overline{\mathrm{Nu}}=\int_{h} \mathrm{Nu} d X$, where $h=L / 2$ is length of thermally active part.

\section{Method of solution}

The governing (2.4)-(2.7) were discretized using the finite volume formulation, with power-law scheme (Patankar [7]). The region of interest was covered with $m$ vertical and $n$ horizontal uniformly spaced grid lines. The numerical solution was true-transient and fully implicit. At each time step the temperature and vorticity distributions were obtained from (2.4) and (2.5), respectively. The resulting set of discretized equations for each variable is solved by a line-by-line procedure, combining the tridiagonal matrix algorithm (TDMA), and the stream function distribution was obtained from (2.6) by using successive over relaxation (SOR) and a known vorticity distribution. The dimensionless time step which yielded convergence for the majority of cases was $\tau=10^{-4}$. An iterative process was employed to find the streamfunction, vorticity, and temperature fields. The process was repeated until the following convergence criterion was satisfied:

$$
\left|\frac{\phi_{n+1}(i, j)-\phi_{n}(i, j)}{\phi_{n+1}(i, j)}\right| \leq 10^{-5} .
$$




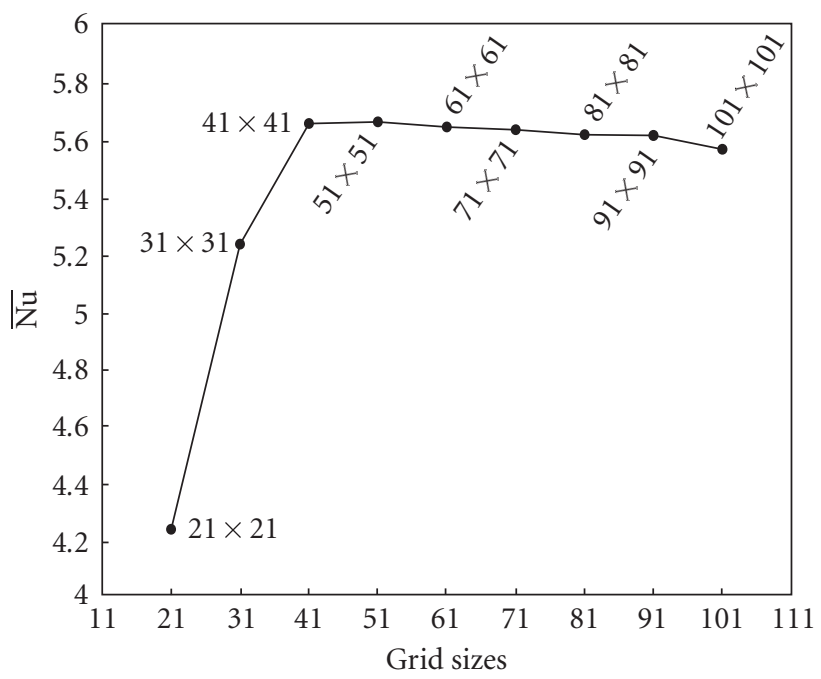

Figure 3.1. Average Nusselt number for different grid sizes.

The overall Nusselt number was also used to develop an understanding of that grid fineness is necessary for accurate numerical simulations. The numerical solutions were done for different grid system from $21 \times 21$ to $101 \times 101$. After $41 \times 41$ grids, there is no considerable change in average Nusselt number, see Figure 3.1. So $41 \times 41$ grid is used to find solutions for different parameters because a grid containing $41 \times 41$ meshes yields satisfactory results.

\section{Result and discussion}

The investigations are carried out for different thermally active locations, Grashof numbers, amplitudes, and periods of time-periodic hot wall temperature. Even though there are nine different thermally active locations considered, the time history of temperature and flow fields is given for the cases, middle part of both thermally active walls, and bottom of the hot wall and top of the cold wall. In Figures 4.1-4.6, the darkened area of side walls indicates the thermally active position. Figures $4.1-4.3$ show the isotherms, streamlines, and velocity vectors for $\mathrm{Gr}=10^{6}, A=0.4, P=1$, and middle-middle thermally active location. In the beginning conduction dominates. For $\tau=0.005$, the isotherms are attracted towards the top corner of the hot wall. It is clearly seen from streamlines and velocity vectors that there exists a small counter acting vertex near the top corner of the hot wall. Further increasing in $\tau=0.01$ the convection mode starts and the clockwise rotating cell occupies the left side half of the cavity. When $\tau=0.05$ the counterclockwise vertex grows and strengthens on the top left corner and the clockwise rotating eddy occupies the remaining part of the cavity. After $\tau=0.1$ there is no change in flow and temperature fields. 
6 Convection in cavities with partially active walls

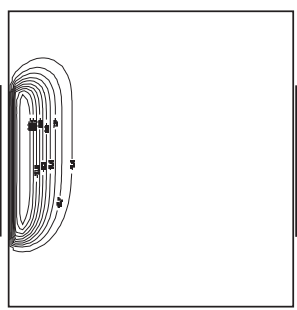

(a) $\tau=0.0025$

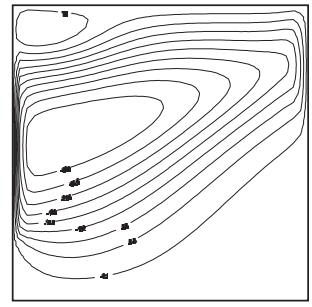

(d) $\tau=0.025$

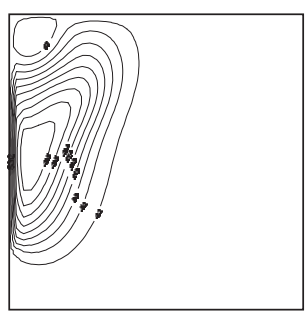

(b) $\tau=0.0075$

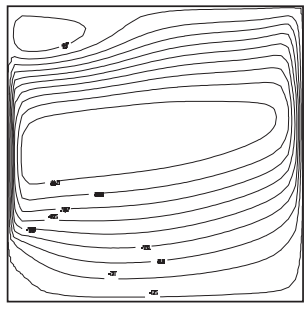

(e) $\tau=0.05$

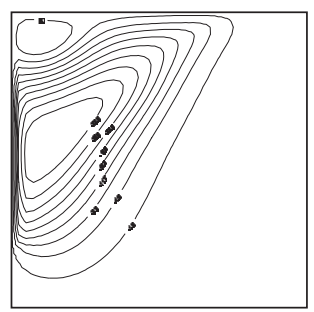

(c) $\tau=0.01$

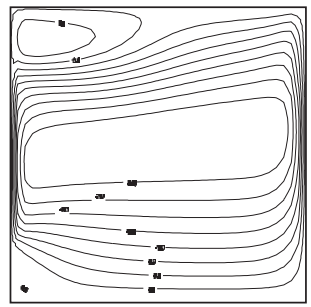

(f) $\tau=0.1$

Figure 4.1. Unsteady-state isotherms, streamlines, and velocity vectors for $\mathrm{Gr}=10^{6}$.

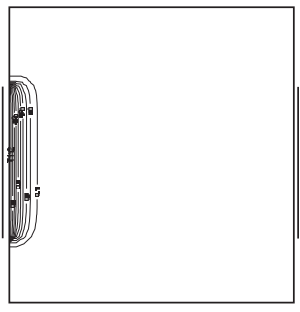

(a) $\tau=0.0025$

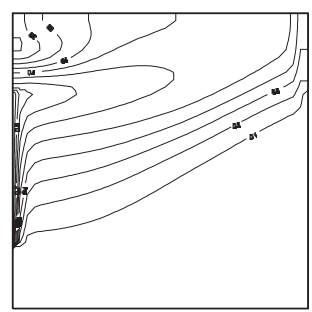

(d) $\tau=0.025$

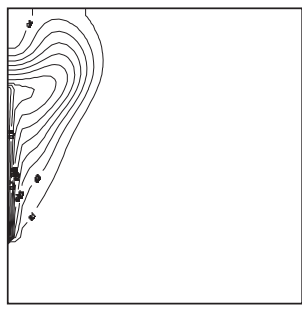

(b) $\tau=0.0075$

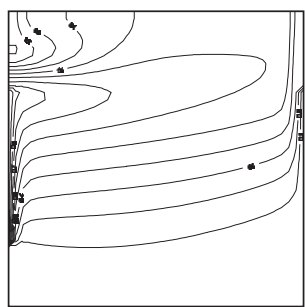

(e) $\tau=0.05$

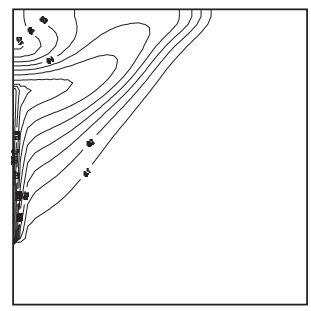

(c) $\tau=0.01$

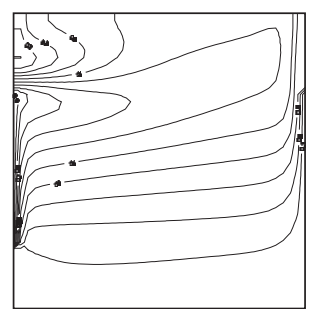

(f) $\tau=0.1$

Figure 4.2. Unsteady-state isotherms, streamlines, and velocity vectors for $\mathrm{Gr}=10^{6}$. 
N. Nithyadevi et al. 7

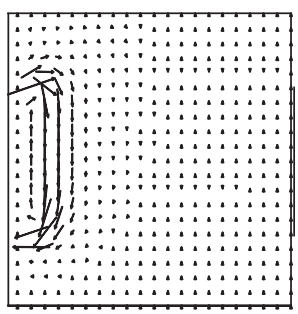

(a) $\tau=0.0025$

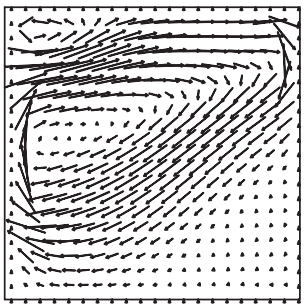

(d) $\tau=0.025$

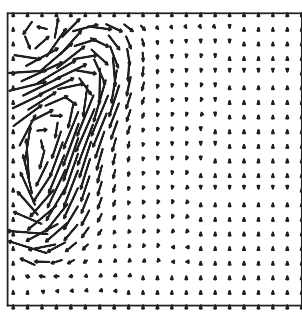

(b) $\tau=0.0075$

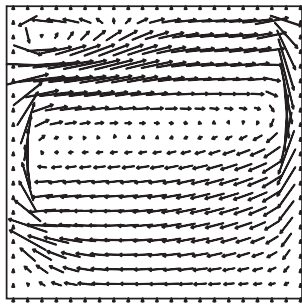

(e) $\tau=0.05$

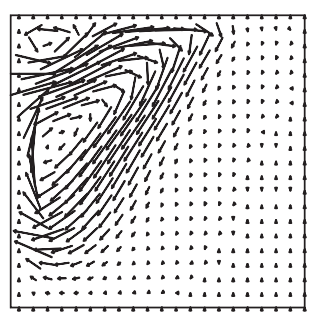

(c) $\tau=0.01$

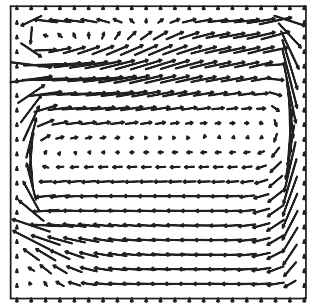

(f) $\tau=0.1$

Figure 4.3. Unsteady-state isotherms, streamlines, and velocity vectors for $\mathrm{Gr}=10^{6}$.

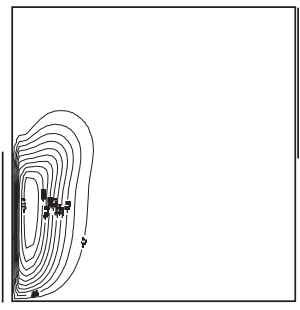

(a) $\tau=0.0025$

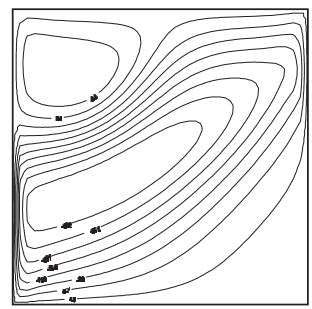

(d) $\tau=0.025$

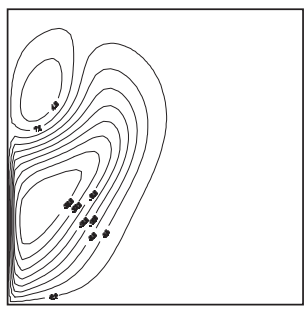

(b) $\tau=0.0075$

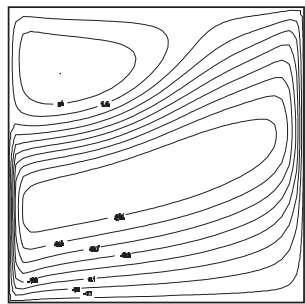

(e) $\tau=0.05$

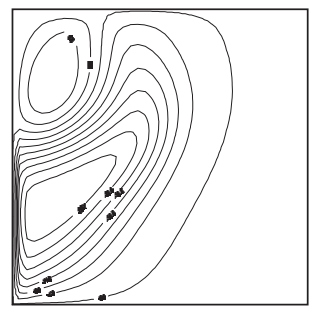

(c) $\tau=0.01$

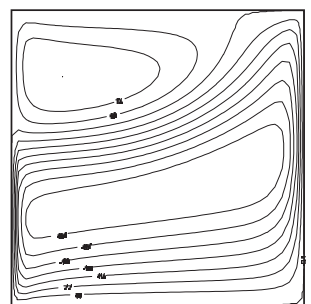

(f) $\tau=0.1$

Figure 4.4. Unsteady-state isotherms, streamlines, and velocity vectors for $\mathrm{Gr}=10^{6}$. 
8 Convection in cavities with partially active walls

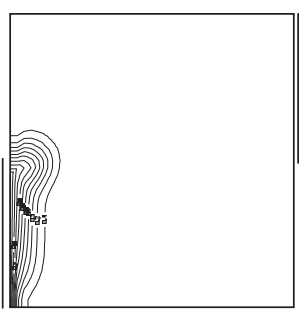

(a) $\tau=0.0025$

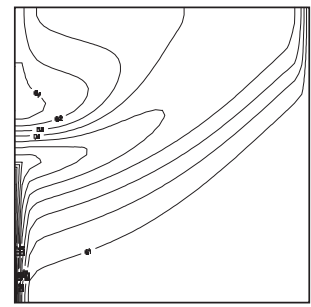

(d) $\tau=0.025$

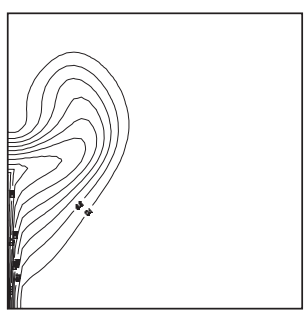

(b) $\tau=0.0075$

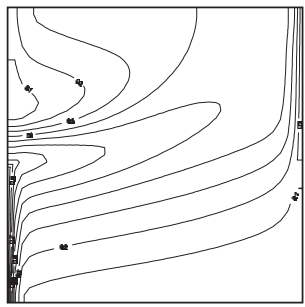

(e) $\tau=0.05$

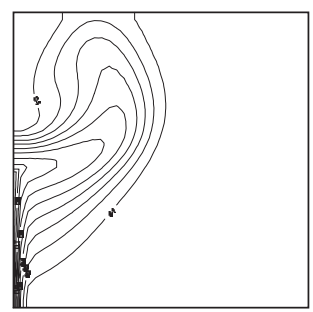

(c) $\tau=0.01$

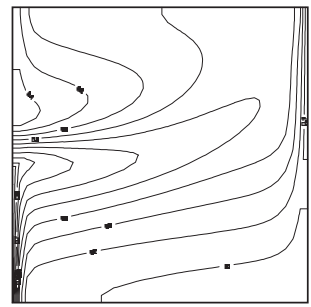

(f) $\tau=0.1$

Figure 4.5. Unsteady-state isotherms, streamlines, and velocity vectors for $\mathrm{Gr}=10^{6}$.

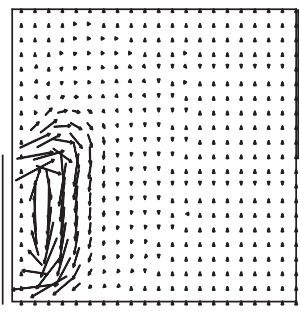

(a) $\tau=0.0025$

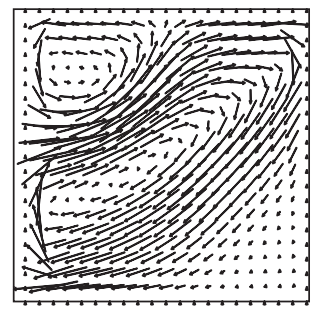

(d) $\tau=0.025$

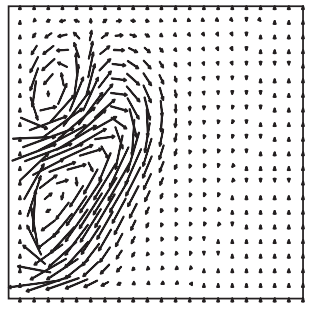

(b) $\tau=0.0075$

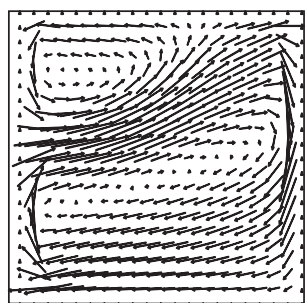

(e) $\tau=0.05$

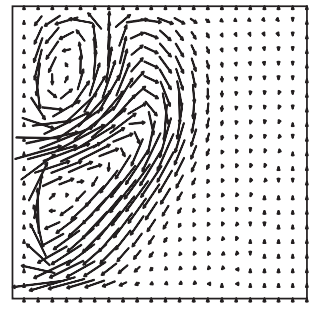

(c) $\tau=0.01$

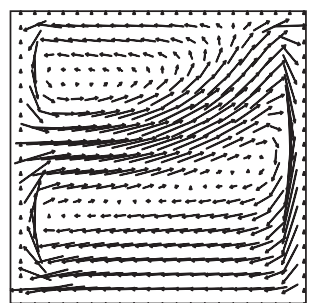

(f) $\tau=0.1$

Figure 4.6. Unsteady-state isotherms, streamlines, and velocity vectors for $\mathrm{Gr}=10^{6}$. 


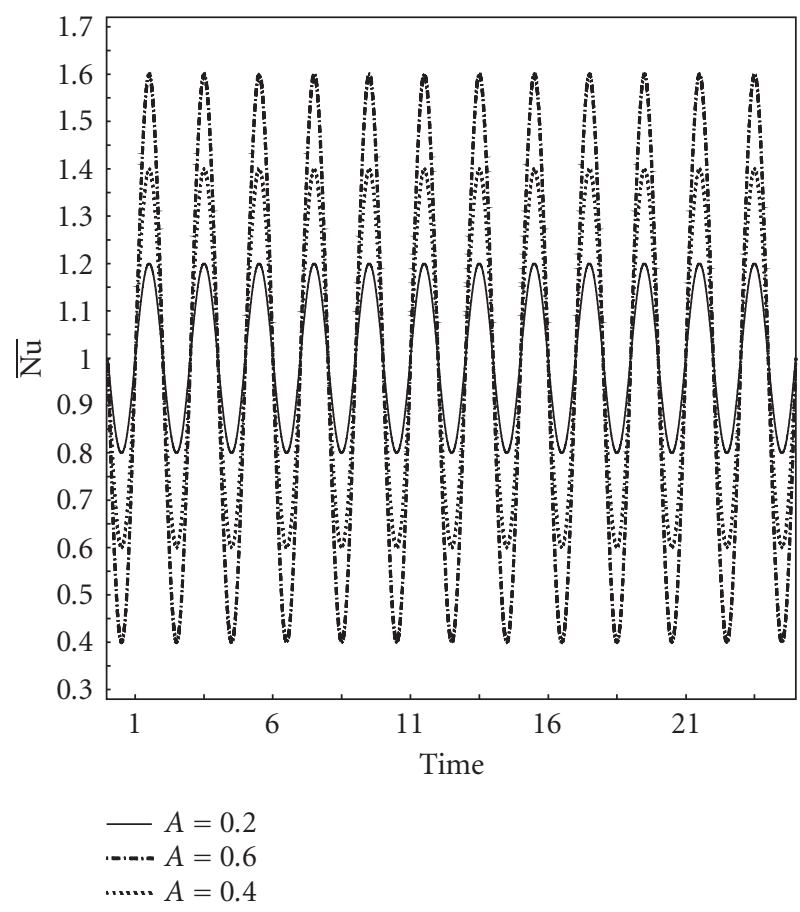

Figure 4.7. Variation of temperature gradient along the hot wall for different amplitude $A, P=1$, and $\mathrm{Gr}=10^{6}$.

The transient results of the heat and flow fields for $\mathrm{Gr}=10^{6}, A=0.4, P=1$, and bottom-top thermally active location are drawn on Figures 4.4-4.6. At the very beginning, $\tau=0.0025$, the left bottom corner of the cavity is active and the remaining portion of the fluid inside the cavity is stagnant. Hence conduction is dominante (Figure 4.4(a)). As time increases a small counter rotating vortex appears on the top left corner and the clockwise cell grows and occupies the majority of the cavity. Further, increasing time ( $\tau=0.01,0.025)$, the clockwise vortex occupies the whole cavity except the top left corner. Still increasing time, the counter-rotating cell near the top left corner grows and strengthen. After that the same behavior was found for all $\tau(\tau \rightarrow \infty)$.

Figure 4.7 shows the variation of the hot wall temperature for different amplitudes $A=0.2,0.4,0.6$ and period $P=1$. These curves clearly indicate the variation in temperature of the hot wall. For $A=0.2$, the maximum and minimum hot wall temperatures are 1.2 and 0.8 , respectively. Similarly for $A=0.4,0.6$, the maximum and minimum hot wall temperatures are 1.4, 0.6 and 1.6, 0.4 , respectively. Variation of hot wall temperature for different periods $P=1,3,5$ and $A=0.4$ is displayed in Figure 4.8. Figure 4.9 shows the time history of the average Nusselt number for different Grashof numbers $\mathrm{Gr}=10^{4}$, $10^{5}, 10^{6}$ with period $P=1$ and amplitude $A=0.4$. Increase in Grashof number increases the average Nusselt number. 


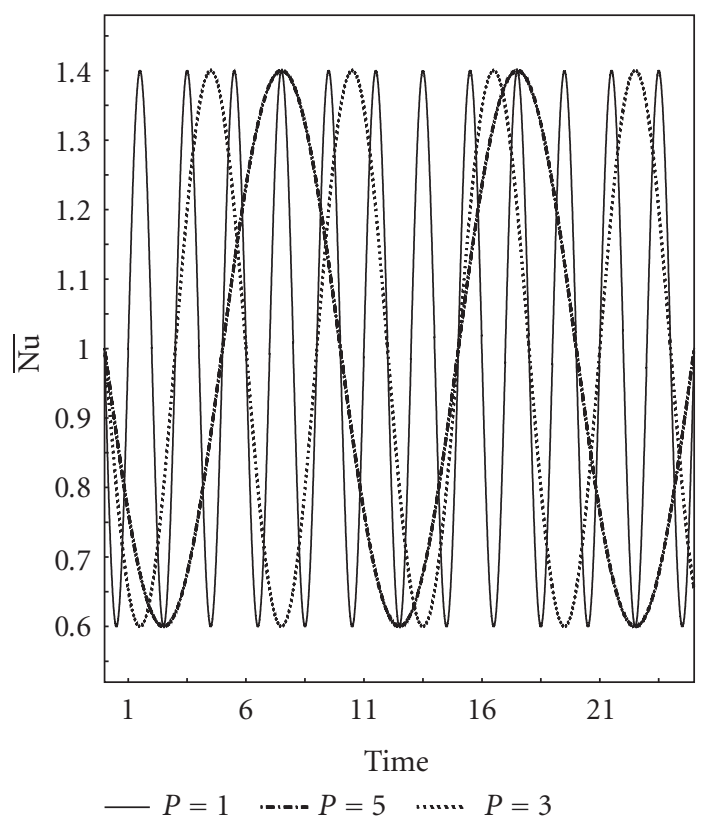

Figure 4.8. Variation of temperature gradient along the hot wall for different period $P, A=0.4$, and $\mathrm{Gr}=10^{6}$.

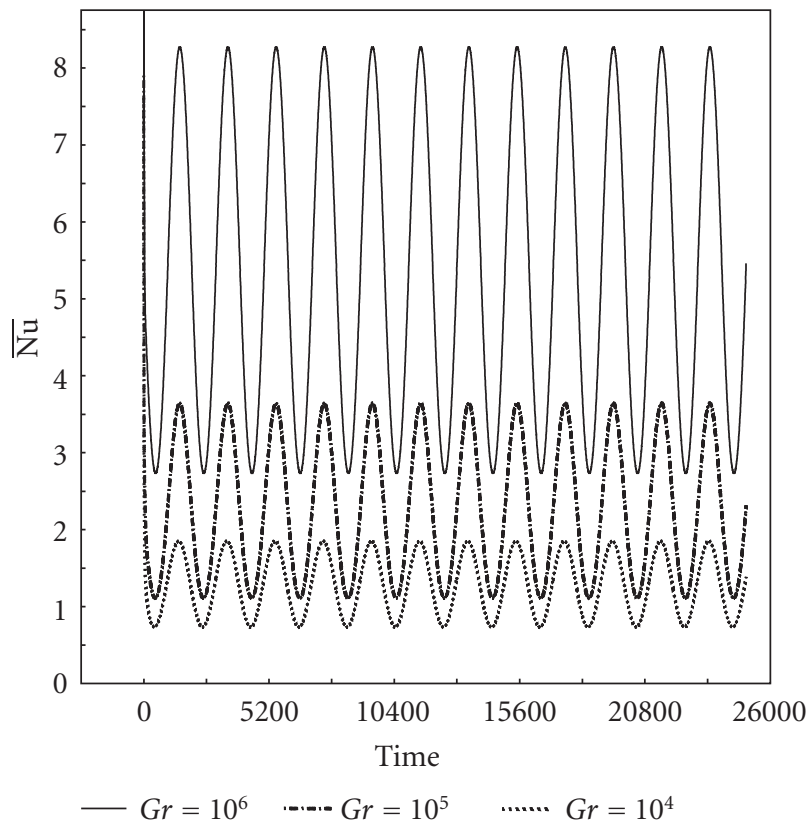

Figure 4.9. Oscillation of average Nusselt number for different Grashof numbers, $P=1$, and $A=0.4$. 


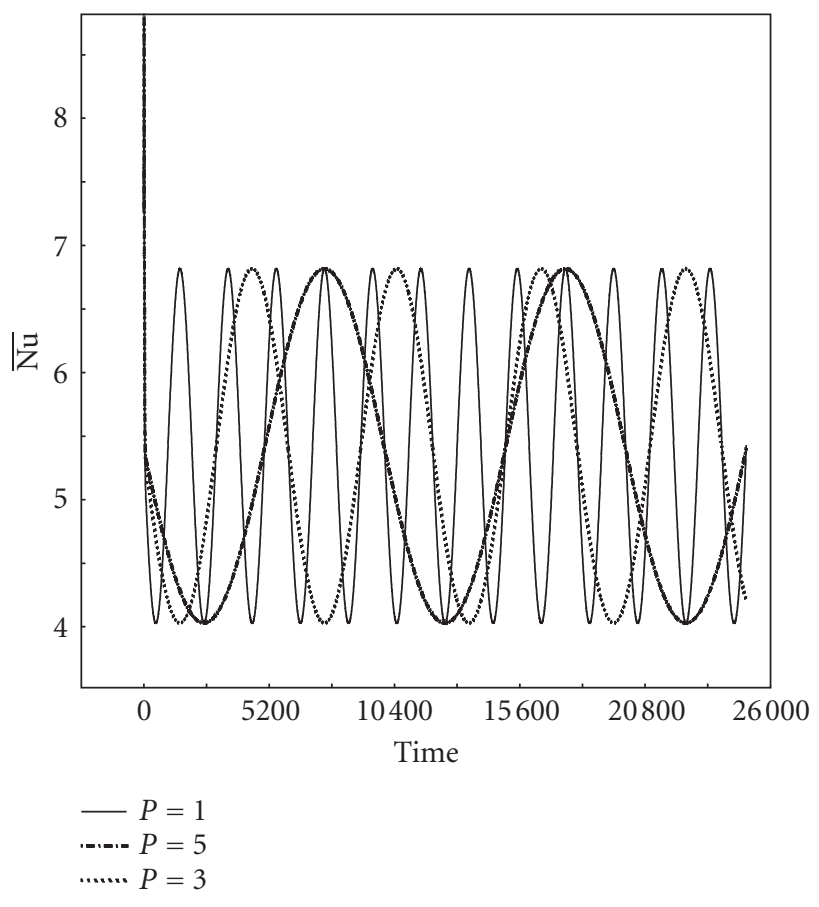

Figure 4.10. Oscillation of average Nusselt number for different period $P, A=0.4$, and $\mathrm{Gr}=10^{6}$.

Figure 4.10 shows the average Nusselt number variation for different periods $P=1,3,5$ and $A=0.2$. The variation of average Nusselt number lies between 6.8 and 4.2 for all values of period after certain time. Figure 4.11 shows the variation of the average Nusselt number versus the amplitude for different periods. For a fixed period (i.e., $P=1$ ) the enhanced heat transfer rate is found for bottom-top thermally active location in Figure 4.11(a). There is no considerable variation in the average Nusselt number for increasing amplitude $A$ and $P=1$. When $P=3$ the heat transfer rate is decreased for increasing the amplitude. Further increasing period $P=5$, the same behavior is observed as in the case of Figure 4.11(a).

The effect of period for different amplitude and Grashof number $\mathrm{Gr}=10^{6}$ is drawn on Figures 4.12(a)-4.12(c). It is clearly seen from these figures that the average Nusselt number behaves nonlinearly as a function of period. The variation among the thermally active location is very small for increasing amplitude. To evaluate how the thermally active location effects the average Nusselt number along the hot wall, average Nusselt number is plotted as a function of Grashof number for $A=0.4$ and $P=1$ in Figure 4.13. When $\mathrm{Gr}<4.0 \times 10^{5}$, heat transfer is to be maximum for the thermally active location at the middle part of the hot wall and the top part of the cold wall, but when $\mathrm{Gr} \geq 4.0 \times 10^{5}$, the thermally active location is at the bottom of the hot wall and cool at the top of the other wall giving better result. For low values of Grashof number $\left(\mathrm{Gr}=10^{4}\right)$ there is 
12 Convection in cavities with partially active walls

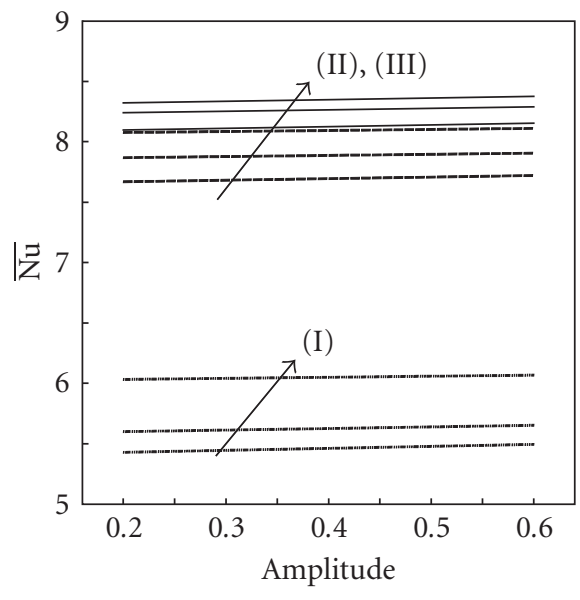

(a) $P=1$

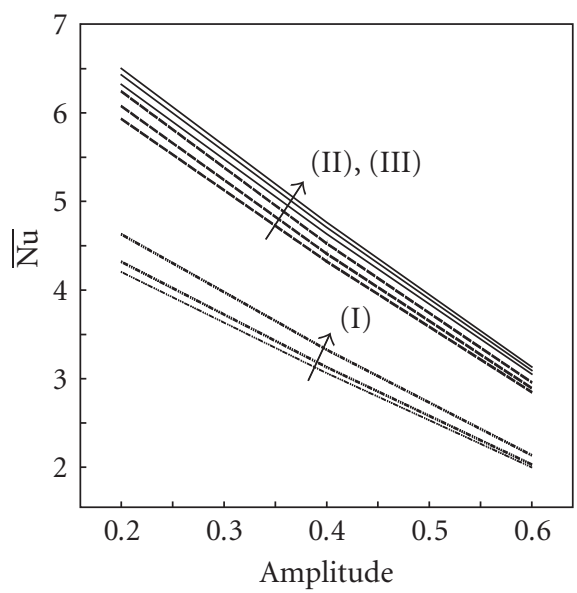

(b) $P=3$

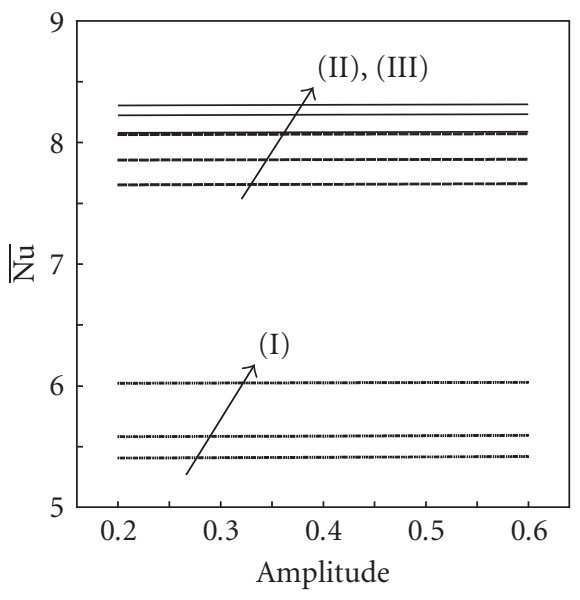

(c) $P=5$

Figure 4.11. Variation of average Nusselt number versus amplitude for different period and $\mathrm{Gr}=10^{6}$.

no variation for change in cold wall location. This is clearly seen from Figure 4.13 that the curves are merged. Increasing Gr values, the difference increases slowly. There is no considerable variation on changing the active parts of the cold wall when the hot wall is fixed at one location.

Figure 4.14 shows the mid-height velocity profiles for $\mathrm{Gr}=10^{6}$ and different thermally active locations. The particle near the vertical walls has higher velocity (curves II) for the middle part thermally active of the left (hot) wall and for all cases of the right (cold) wall than the other two locations of thermally active of left wall (curves I and III). The variation of the transient local Nusselt number with time along the hot wall of the cavity 
N. Nithyadevi et al. 13

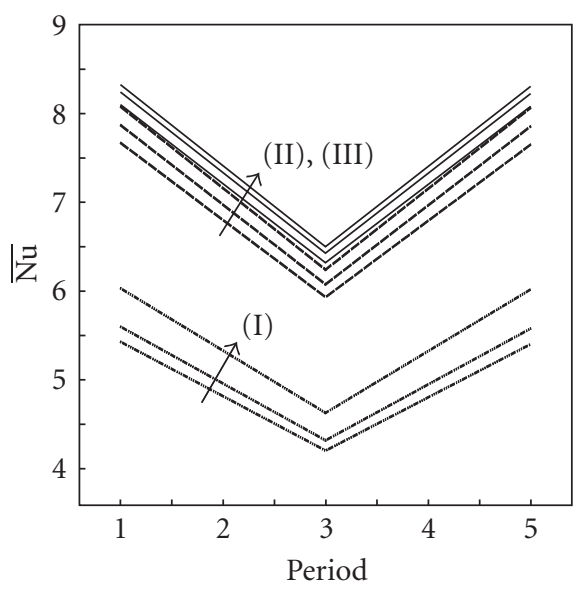

(a) $A=0.2$

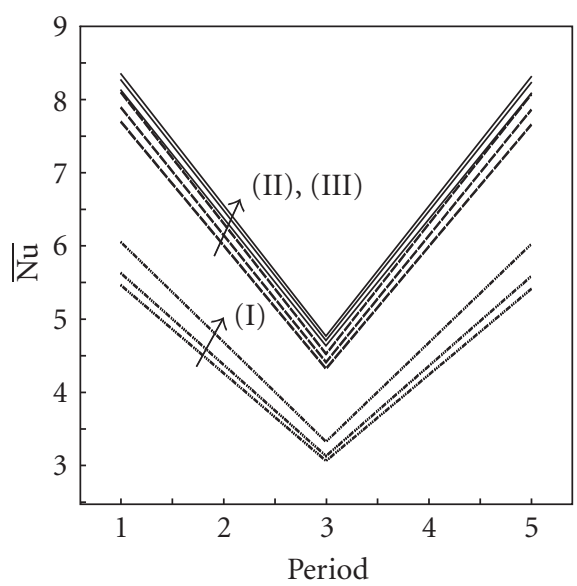

(b) $A=0.4$

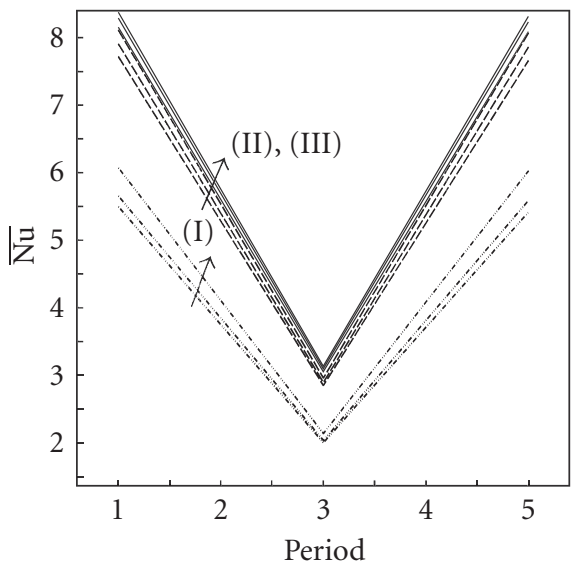

(c) $A=0.6$

Figure 4.12. Variation of average Nusselt number versus period for different amplitude and $\mathrm{Gr}=10^{6}$.

at different position is presented in Figure 4.15 for $\mathrm{Gr}=10^{4}-10^{6}$. It is seen that the local Nusselt number decreases for a short time and then increase to reach the steady state value.

\section{Conclusion}

It is observed that heat transfer is enhanced when thermally active location is at the middle of the hot wall for $\mathrm{Gr}<4.0 \times 10^{5}$ and at the bottom of the hot wall for $\mathrm{Gr} \geq 4.0 \times 10^{5}$, respectively. Increasing Grashof number, heat transfer rate increases. Circulation rate of 
14 Convection in cavities with partially active walls

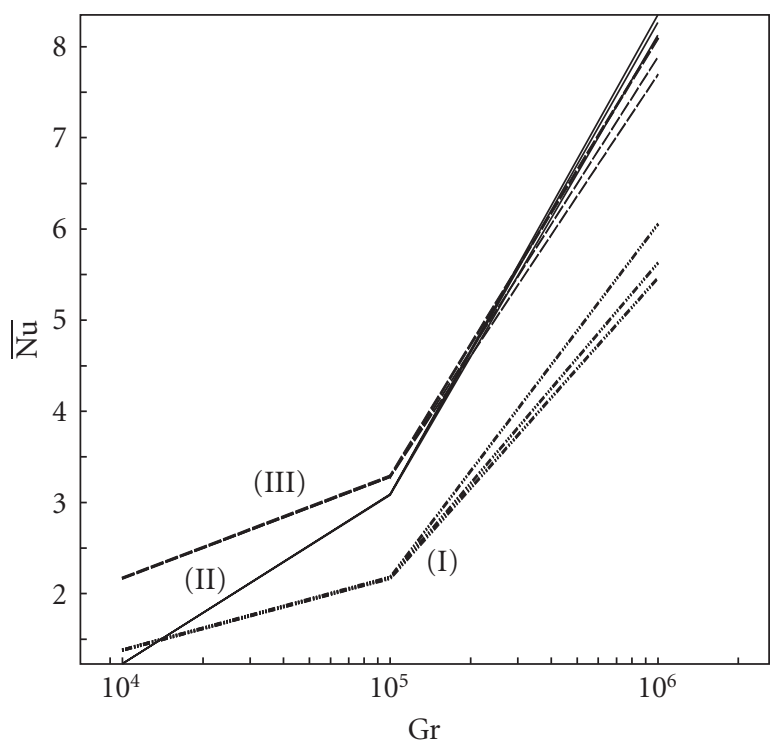

Figure 4.13. Average Nusselt number for different Grashof numbers, $P=1$, and $A=0.4$. (I) Top active hot wall, (II) middle active hot wall, (III) bottom active hot wall.

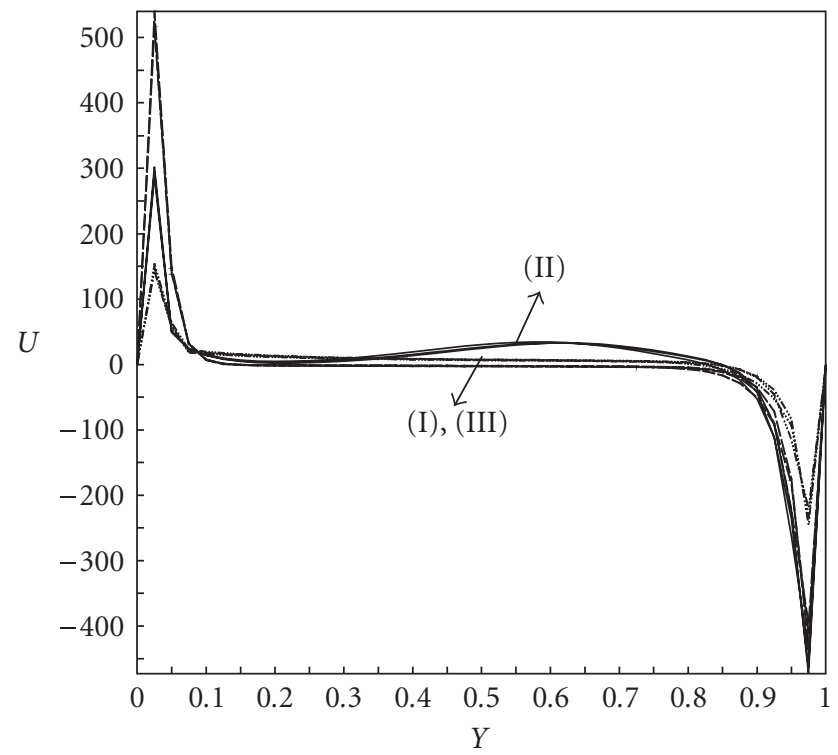

Figure 4.14. Vertical velocity profiles at mid-hight for $\mathrm{Gr}=10^{6}, P=1$, and $A=0.4$. 


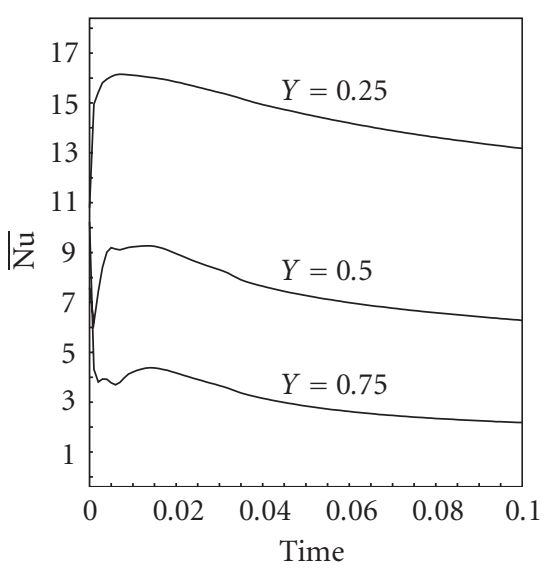

(a) $\mathrm{Gr}=10^{4}$

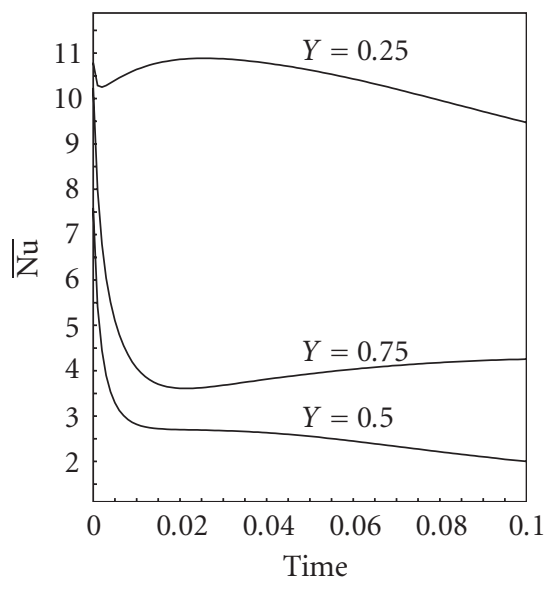

(b) $\mathrm{Gr}=10^{5}$

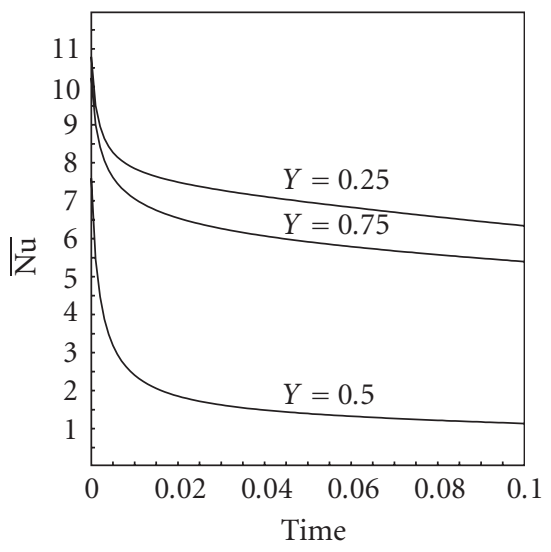

(c) $\mathrm{Gr}=10^{6}$

Figure 4.15. Variation of the transient local Nusselt number for $P=1$ and $A=0.4$.

the eddy is increased when increasing Grashof number. There is no considerable variation due to changing the thermally active parts of the cold wall when the thermally active location of the hot wall is fixed. The average Nusselt number behaves nonlinearly as a function of period. The heat transfer increases for increasing amplitude, $P=1,5$. When $P=3$, the average heat transfer rate is decreased.

\section{References}

[1] B. V. Antohe and J. L. Lage, Amplitude effect on convection induced by time-periodic horizontal heating, International Journal of Heat and Mass Transfer 39 (1996), no. 6, 1121-1133.

[2] D. W. Crunkleton, R. Narayanan, and T. J. Anderson, Numerical simulations of periodic flow oscillations in low Prandtl number fluids, International Journal of Heat and Mass Transfer 49 (2006), no. 1-2, 427-438. 
16 Convection in cavities with partially active walls

[3] G. B. Kim, J. M. Hyun, and H. S. Kwak, Enclosed Buoyant convection with internal heat generation under oscillating sidewall temperature, ASME Journal of Heat Transfer 124 (2002), no. 3, 577580 .

[4] D. Kuhn and P. H. Oosthuizen, Unsteady natural convection in a partially heated rectangular cavity, ASME Journal of Heat Transfer 109 (1987), 789-801.

[5] E. K. Lakhal, M. Hasnaoui, and P. Vasseur, Numerical study of transient natural convection in a cavity heated periodically with different types of excitations, International Journal of Heat and Mass Transfer 42 (1999), no. 21, 3927-3941.

[6] A. Pantokratoras, Laminar natural convection in water near the density extremum along a vertical plate with sinusoidal surface temperature variation, Acta Mechanica 172 (2004), no. 3-4, 211218.

[7] S. V. Patankar, Numerical Heat Transfer and Fluid Flow, Hemisphere, Washington, DC; McGrawHill, New York, 1980.

[8] N. H. Saeid and A. A. Mohamad, Periodic free convection from a vertical plate in a saturated porous medium, non-equilibrium model, International Journal of Heat and Mass Transfer 48 (2005), no. $18,3855-3863$.

N. Nithyadevi: Department of Mathematics and UGC-DRS Centre for Fluid Dynamics, Bharathiar University, Coimbatore 641 046, India

E-mail address: nithyadevin@yahoo.co.in

P. Kandaswamy: Department of Mathematics and UGC-DRS Centre for Fluid Dynamics, Bharathiar University, Coimbatore 641 046, India

E-mail address: pgkswamy@yahoo.co.in

S. Sivasankaran: Department of Mathematics and UGC-DRS Centre for Fluid Dynamics, Bharathiar University, Coimbatore 641 046, India

E-mail address: siva_sd2000@yahoo.com 


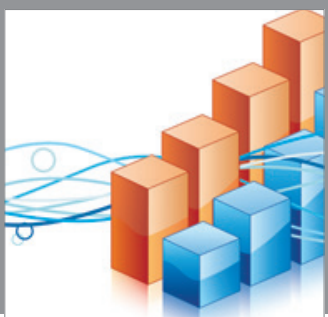

Advances in

Operations Research

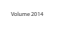

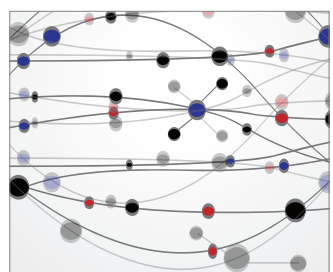

\section{The Scientific} World Journal
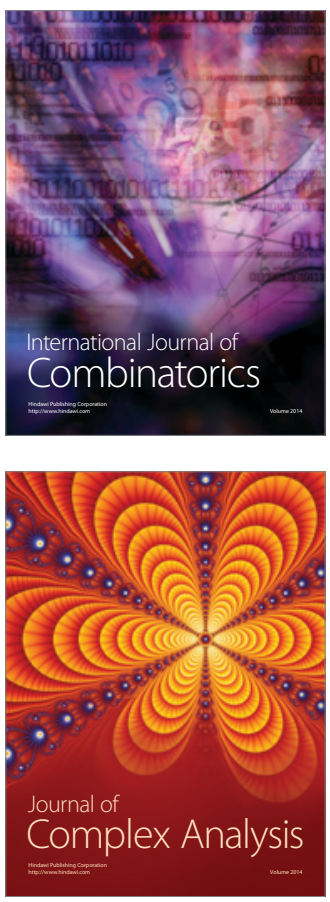

International Journal of

Mathematics and

Mathematical

Sciences
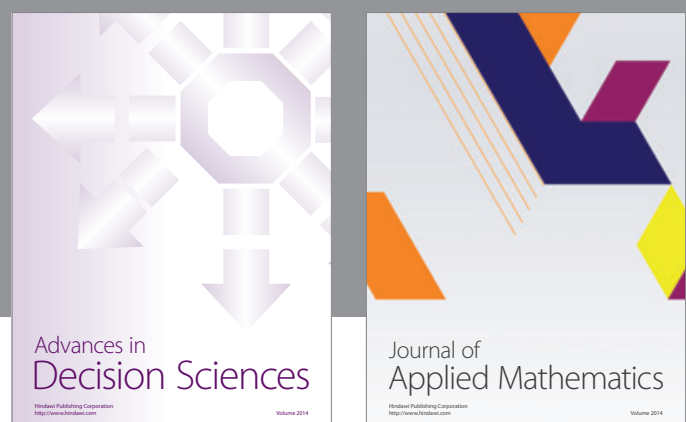

Journal of

Applied Mathematics
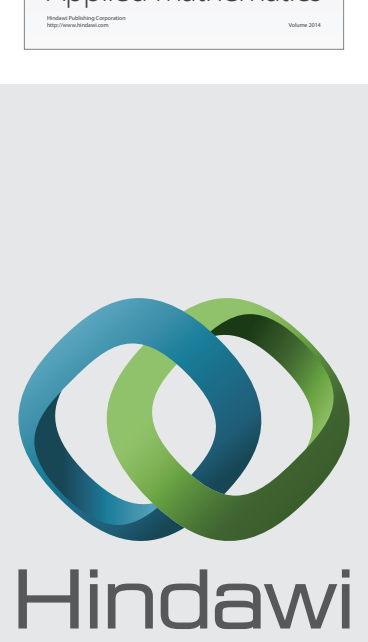

Submit your manuscripts at http://www.hindawi.com
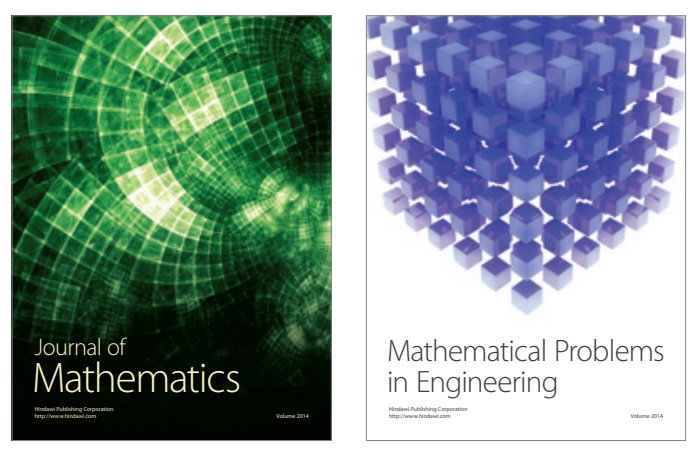

Mathematical Problems in Engineering
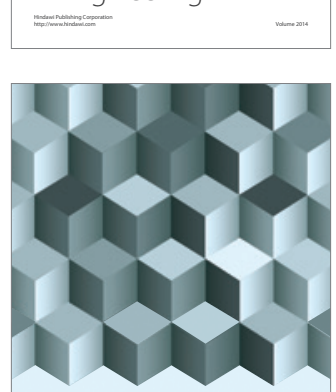

Journal of

Function Spaces
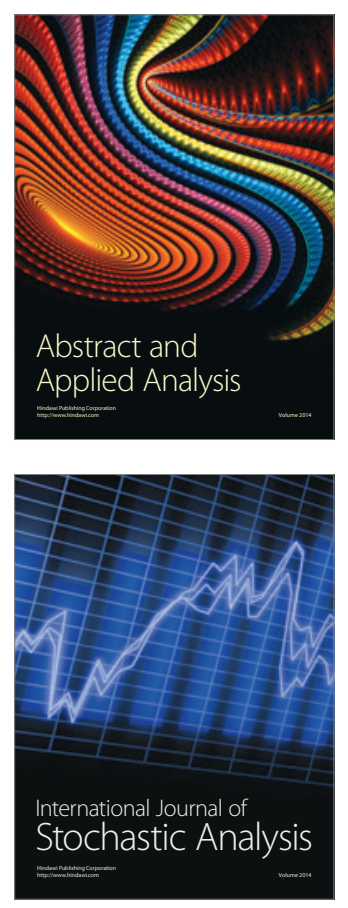

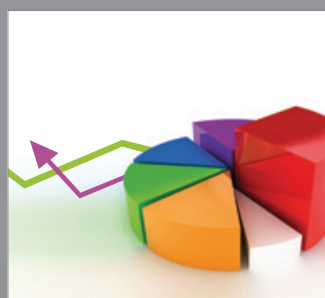

ournal of

Probability and Statistics

Promensencen
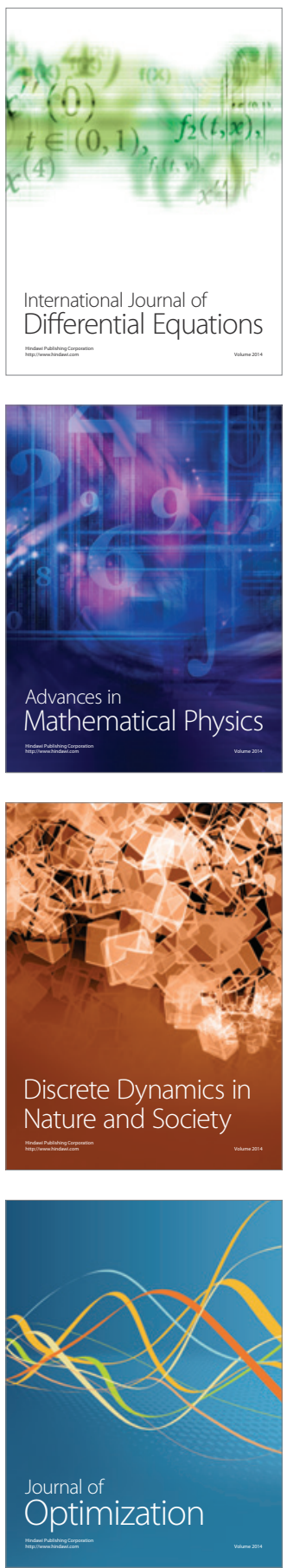